\title{
Pragmatism and Theories of Emergence
}

\section{Justificación Y Noción De Verdad}

\section{Mónica Gómez Salazar}

\section{(2) OpenEdition}

\section{Journals}

Edición electrónica

URL: http://journals.openedition.org/ejpap/1707

DOI: 10.4000/ejpap.1707

ISSN: 2036-4091

\section{Editor}

Associazione Pragma

Referencia electrónica

Mónica Gómez Salazar, « Justificación Y Noción De Verdad », European Journal of Pragmatism and American Philosophy [Online], XI-2 | 2019, Online since 24 December 2019, connection on 15 June 2020. URL : http://journals.openedition.org/ejpap/1707 ; DOI : https://doi.org/10.4000/ejpap. 1707

Este documento fue generado automáticamente el 15 junio 2020

\section{(c) $($ i) $(9)$}

Author retains copyright and grants the European Journal of Pragmatism and American Philosophy right of first publication with the work simultaneously licensed under a Creative Commons Attribution-

NonCommercial-NoDerivatives 4.0 International License. 


\title{
Justificación Y Noción De Verdad
}

\author{
Mónica Gómez Salazar
}

\section{NOTA DEL AUTOR}

Investigación realizada gracias al Programa UNAM-DGAPA-PAPIIT IN403017 ‘Sofística y Pragmatismo.'

\section{Contrapropuesta rortyana a la noción de verdad}

1 La cuestión de si existe algún conjunto de creencias comunes a todos los seres humanos tiene poco interés, nos dice Rorty, si no es en relación con una visión inclusivista de la comunidad humana. Una visión de comunidad como ésta se enorgullece más de los distintos tipos de gente a los cuales da la bienvenida, que de la firmeza con que mantiene alejados a los extraños. Para este pensador el término "política democrática" es sinónimo del intento de realizar semejante comunidad (Rorty 2000: 79).

2 Siguiendo esta idea, Rorty explica que algunos de los filósofos interesados en la política democrática admiten las siguientes tres premisas: 1) El deseo de verdad es un deseo universal, 2) la verdad es correspondencia con la realidad y 3) la realidad tiene una naturaleza intrínseca. Tras la aceptación de estas tres premisas dichos filósofos argumentan que la verdad es una y que el interés humano universal por la verdad proporciona suficientes motivos como para crear una comunidad inclusivista. De acuerdo con este argumento, los sujetos que descubran la verdad única tendrán más cosas en común para compartir y por consiguiente, sus miembros serán más tolerantes e inclusivistas.

Desde la posición de Rorty, es posible defender una política democrática y negar al mismo tiempo cualquiera de las tres premisas anteriores. La mejor forma de presentar aquello que los filósofos han descrito como deseo universal de verdad es describiéndolo como deseo universal de justificación. De suerte que una de las diferencias que habría entre verdad y justificación sería la diferencia entre lo que no se puede reconocer y lo 
que puede ser reconocido. Es decir, no podemos llegar a saber con certeza si una creencia es verdadera o no, pero sí podemos llegar a no tener objeción residual en su contra.

4 La noción de verdad como anhelo universal que lleva a ciertos filósofos a insistir en que debemos evitar el "contextualismo" y el "relativismo," es considerada como un anhelo de verdad que nos lleva a pagar el precio de la irrelevancia práctica. De ahí que según este autor, la verdad no puede ser relevante para la política democrática y termine ciñéndose a la justificación (Rorty 2000: 80-2). La importancia de la justificación residiría en que, si bien suele ser provisional, puesto que antes o después aparecerán nuevas objeciones en contra de la creencia justificada, puede ser reconocida.

5 Según esta postura, no disponemos de ningún terreno neutral desde el que sea posible defender la política democrática. Lo importante en el ser humano es su capacidad para ser miembro de una comunidad inclusivista y no su capacidad de conocimiento para captar la verdad.

6 Para Rorty, una creencia puede estar justificada pero no ser verdadera de modo que no hace falta contar con una teoría filosófica sobre la naturaleza de la verdad; el contraste que existe entre verdad y justificación es de orden práctico. Entre los diversos usos de la palabra "verdadero," el único que no puede ser eliminado con facilidad de nuestra práctica lingüística es el uso de advertencia. Este uso de advertencia es utilizado para contrastar audiencias poco informadas con audiencias mejor informadas y, más generalmente, para contrastar audiencias pasadas con audiencias futuras.

En contextos no filosóficos, el sentido de contrastar justificación con verdad es, simplemente, recordarnos que pueden haber objeciones (a causa de la aparición de nuevos datos, nuevas hipótesis explicativas más ingeniosas, cambios en el vocabulario empleado para describir los objetos que se discuten) que no hayan advertido ninguna de las audiencias para las cuales la creencia en cuestión estaba hasta entonces justificada. (Rorty 2000: 87)

7 La premisa fundamental de esta propuesta es que solamente podemos trabajar por lo que podemos reconocer. Éste es un corolario del principio de James que afirma que para que valga la pena discutir una diferencia, ésta tiene que ser relevante en el orden práctico. Es decir, supongamos que tenemos dos definiciones filosóficas, o proposiciones o máximas, que aparentemente se contradicen y que son objeto de discusión entre los sujetos. Si suponiendo la verdad de una no es posible prever ninguna consecuencia práctica concebible para nadie en ningún momento o lugar, que sea distinta de lo que puede preverse si uno supone la verdad de la otra, en tal caso la diferencia entre las dos proposiciones no es una verdadera diferencia; tan sólo es una distinción aparente y verbal que no vale la pena discutir.

La actitud pragmatista adecuada hacia la verdad, según Rorty, puede resumirse como sigue: es tan poco necesario tener una teoría filosófica sobre la naturaleza de la verdad, o sobre el significado de la palabra "verdadero," como tener una teoría filosófica sobre la naturaleza del peligro o sobre el significado de la palabra "peligro." La razón principal de que en nuestro lenguaje exista una palabra como 'peligro' es advertir a la gente que es imposible que haya previsto todas las consecuencias de las acciones que se propone llevar a cabo. Es decir, los pragmatistas que entienden las creencias como hábitos de acción, creen que el uso de advertencia de la palabra "verdadero," lejos de corresponder a la realidad, se utiliza para tener presente que otros sujetos, en circunstancias distintas, enfrentándose a audiencias futuras, podrían ser incapaces de 
justificar una creencia que hasta ese momento han justificado con éxito ante todas las audiencias con las que se han encontrado. Ésta es la concepción pragmatista de la función de la palabra "verdadero."

9 En lo que respecta a la pretensión de validez universal de las creencias, muchas pretensiones de validez son formuladas por personas que desearían defender sus afirmaciones ante audiencias distintas de aquella a la que se dirigen en ese momento. Sin embargo, "[...] decir 'voy a tratar de defender esto frente a quien sea' suele ser propio de una actitud encomiable. Pero decir 'puedo defender esto con éxito frente a quien sea' es una tontería" (Rorty 2000: 93). Con todo, llegar a distinguir entre si cierta afirmación depende del contexto o es universal, si cierta creencia es correcta contextualmente o universalmente, el favorecer una alternativa y no otra, no supone ninguna relevancia de orden práctico.

Habermas, por ejemplo, distingue entre aquellas prácticas de justificación orientadas a pretensiones de verdad, de aquellas otras prácticas reguladas meramente por convención social, como las prácticas morales y políticas (Habermas 1998: 77). Para Rorty las prácticas no trascienden, no se extienden fuera de la convención social, por el contrario, están reguladas por determinadas convenciones sociales, de modo que no hay distinción entre práctica social y algo que, como límite, esté más allá de esas prácticas.

Desde la postura que defendemos, es condición necesaria que las creencias sean justificadas en relación con la noción de verdad. Esta noción, correlativa a la noción de realidad, es la que permite que el mundo en el que vivimos y que construimos por medio de nuestras prácticas no sea un mundo que se constituye debido a decisiones arbitrarias, insuficientemente fundamentadas. Siguiendo esta idea, el justificar las creencias y contar con razones que las aseguren como verdaderas no significa acceder a Una Verdad Universal de El Mundo. La verdad de las creencias podría ser entendida como una verdad a la que se accede a través de las razones de los sujetos, entendida como una noción de verdad epistémica y social, no separable de los sujetos. En este sentido, las creencias serían verdaderas en nuestro mundo y accederíamos a la verdad de las proposiciones por medio de nuestras justificaciones, las cuales son falibles. Así, los sujetos de una comunidad pueden tener una garantía a la medida humana de que ciertas prácticas serán exitosas, en tanto que cuenten con razones suficientes que garanticen que sus acciones (al tratar de incidir en esa realidad, para actuar sobre ella y transformarla) acertarán la realidad. La importancia de la noción de verdad reside en que, a pesar de que las creencias de las diferentes comunidades epistémicas sean justificadas mediante razones que pueden ser corregibles en otro momento histórico y en relación con otra comunidad, podemos sin embargo, mantener la pretensión de que esas creencias lejos de ser arbitrarias, están restringidas por la realidad y son guías de acciones acertadas. Para esto requerimos una noción de verdad. Habría que reconocer, sin embargo, que hasta aquí no hemos más que ofrecido una explicación sobre los criterios de verdad y sobre las justificaciones. Aunque sostengamos con pragmatistas como James (2011) y Schiller que hay una realidad que se infiere, que restringe nuestras prácticas, mostrándonos cómo es que éstas son o no acertadas, no accedemos a esa realidad más que a través de nuestras justificaciones y marcos de presupuestos.

De acuerdo con Schiller, debe ser admitido que aquello que consideramos un hecho es porque ha pasado por un proceso de selección que aunque esté guiado por los resultados o consecuencias que confirmen o refuten una hipótesis, también hay que 
aceptar que este proceso es en buena medida falible, pues depende de nuestro limitado alcance y perspectiva (Schiller 1907: 185-8).

\section{Los mundos entendidos como construcciones onto- epistemológicas plurales.}

13 Partimos de la idea de que los sujetos estructuran el mundo o realidad a través de alguno de los diversos marcos conceptuales. Los marcos conceptuales son construcciones sociales que se construyen, sostienen y transforman como resultado de las interacciones o prácticas sociales de los sujetos. Estos marcos son condiciones de posibilidad para tener conceptos, creencias, lenguaje, conocimientos, normas y valores que los seres humanos necesitamos y usamos en nuestra relación cognoscitiva con el mundo (Olivé 1999: 135-42). El mundo en el que los integrantes de una comunidad viven depende epistémica y ontológicamente de esos marcos. Por ello, los sujetos al estructurar la realidad desde marcos conceptuales distintos entienden el mundo de otra manera, pero también viven en otro mundo. Así, en un sentido fuerte, lo que cuenta como mundo para una comunidad de sujetos depende de su lenguaje, conocimientos, creencias, normas y valores. Desde esta posición, que se basa en el realismo interno de Hilary Putnam, los sujetos construyen, al menos parcialmente, el mundo en el que viven, y en este sentido, también son en algún grado responsables del mundo que constituyen en sus prácticas sociales.

Esta postura onto-epistemológica se opone a las tesis del realismo metafísico.

15 Siguiendo a Quintanilla (1994: 22), el realismo metafísico puede resumirse en las siguientes tesis:

1. El mundo o realidad existe independientemente de nuestros conceptos y representaciones.

2. El mundo o realidad está compuesto de cosas, propiedades, y hechos que existen independientemente de que nosotros los conozcamos o no.

3. Una representación correcta del mundo refiere a los objetos realmente existentes en

él, y describe las propiedades que los objetos realmente tienen.

4. Existe una representación completa del mundo tal como es en sí mismo.

La primera tesis debe ser admitida por cualquier postura realista. Esta tesis reconoce la existencia del mundo como real y no como una mera creación nuestra. La segunda tesis establece que ese mundo o realidad independiente de nuestros conceptos ya está estructurada por cosas, propiedades y hechos que existen independientemente de que los conozcamos o no. De modo que, como indica la tercera tesis, una representación correcta del mundo sería aquella que se corresponda con los objetos y hechos que existen en él. Según la cuarta tesis, consideremos que si el mundo es en sí mismo de una determinada manera, ya está estructurado por objetos y hechos (tesis 2) y si la verdad en tanto correspondencia implica una correlación entre las representaciones y los objetos y hechos (tesis 3), entonces de acuerdo con el realismo metafísico, sólo puede haber una manera correcta y completa de describir ese mundo. Es decir, si el mundo tiene una estructura ontológica determinada, sólo puede admitir una manera correcta de ser descrito en cada uno de sus aspectos. Según esta postura, si juntáramos todas las descripciones parciales verdaderas del mundo, tendríamos una descripción completa y verdadera del mismo (Pérez 1993). 
17 Así, no tenemos buenas razones para suponer la existencia de una realidad ya estructurada, y tampoco tenemos buenas razones para suponer que podemos conocerla, de ahí que no compartamos la respuesta del realismo metafísico al problema del conocimiento del mundo. De ser así, habría que aceptar la existencia de un único marco conceptual correcto, ya determinado, lo que significaría admitir que hay un único conjunto de creencias, lenguaje, conocimientos, normas y valores válidos gracias a los cuales de manera universal y definitiva los seres humanos podríamos conocer el mundo o realidad.

18 El realismo interno no niega la existencia de una realidad independiente de nuestros marcos conceptuales y de los sujetos cognoscentes, pero sostiene que el mundo o realidad no existe independientemente de nuestros marcos conceptuales. Esta relación entre la realidad de los hechos construidos a partir de los marcos conceptuales y la realidad desde la cual se construyen esos hechos, no debe entenderse como si se tratase de dos realidades. Los hechos y los objetos son reales porque son parte de la realidad independiente, se construyen a partir de ella, pero son construidos como hechos y objetos en relación con los marcos conceptuales. La realidad pues, depende gnoseológicamente y ontológicamente de esos marcos.

Putnam dirá que "los 'objetos' no existen independientemente de los esquemas conceptuales" (Putnam 1981: 52). De ahí que el realismo interno sostenga que una postura realista no es incompatible con la relatividad conceptual, "se puede ser al mismo tiempo un realista y un relativista conceptual" (Putnam 1994: 61).

Por ejemplo, nos dice Putnam, pensemos en un mundo con 3 individuos: individuo 1, individuo 2 e individuo 3. La respuesta a la pregunta ¿cuántos objetos hay en ese mundo?, dependerá de lo que nosotros entendamos por "objeto." Si entendemos por "objeto" lo mismo que por "individuo" diremos que hay 3 objetos. El objeto A constituido por el individuo 1, el objeto B constituido por el individuo 2, y el objeto C constituido por el individuo 3 .

21 Sin embargo, si para entender lo que es un "objeto" consideramos la lógica mereológica, un "objeto" podría estar constituido no sólo por cada uno de los individuos, sino también por las sumas que puedan hacerse de ellos. Así, el objeto A puede estar constituido por el individuo 1, el objeto B por el individuo 2, el objeto C por el individuo 3, el objeto D por la suma del individuo 1 más el individuo 2, el objeto $\mathrm{E}$ por la suma del individuo 1 más el individuo 3, el objeto $\mathrm{F}$ por la suma del individuo 2 más el individuo 3 y finalmente, el objeto $G$ puede estar constituido por la suma del individuo 1 más el individuo 2 más el individuo 3.

Objeto $\mathrm{A}=1$

Objeto $B=2$

Objeto $C=3$

Objeto $\mathrm{D}=1+2$

Objeto $\mathrm{E}=1+3$

Objeto $\mathrm{F}=2+3$

Objeto $G=1+2+3$

La diferencia consiste en cómo se entiende el concepto de "objeto." En el mundo de la primera noción de "objeto" hay 3 objetos reales, y en el mundo de las sumas mereológicas hay 7 objetos también reales constituidos por 3 individuos relacionados de distintas maneras. De suerte que no hay nada independiente a los dos mundos que indique cuál es la respuesta correcta a la pregunta ‘¿cuántos objetos hay?' “[...] las 
nociones de objeto y existencia, tienen una multitud de diferentes usos [...]" (Putnam 1994: 63) y no una concepción absoluta.

El realismo interno sostiene que el planteamiento de conocer la realidad en sí misma carece de sentido porque, aunque "podemos y debemos insistir en que existen hechos que están allí para ser descubiertos y no meramente legislados por nosotros," esto ocurre cuando ya estamos relacionados con la realidad por medio de un marco conceptual. "Hablar de 'hechos' sin antes especificar qué lenguaje se usará, es hablar de nada” (Putnam 1990: 175).

24 Para el realismo interno los hechos y los objetos se construyen en relación con la realidad según los intereses y marcos conceptuales con los que los sujetos estamos familiarizados. Como señala Olivé, al aplicarse un marco conceptual a la realidad se establece el contexto relevante con respecto al cual se construyen las propiedades de los hechos y objetos, esas propiedades serán las que determinen la identidad de éstos en tanto los objetos y hechos que son. Es en este sentido que los objetos y hechos sólo existen en relación con los marcos conceptuales. Si la realidad no fuera estructurada desde los marcos conceptuales que posibilitan la construcción de contextos relevantes, los hechos y objetos no podrían existir (Olivé 1996: 190). En palabras de Sosa: “[...] cuando afirmamos que hay ciertas cosas con ciertas propiedades, nuestra afirmación debe ser considerada como relativa a un lenguaje particular y a un esquema conceptual en particular" (Sosa 1992: 72).

En el ejemplo expuesto, los objetos A, B y C realmente existen en ese mundo, es decir, no son meras creaciones mentales de los sujetos. Desde esta perspectiva no hay pérdida de lo real, el marco conceptual adoptado es uno de los marcos posibles.

Desde el punto de vista del marco de las sumas mereológicas, los objetos también forman parte del mundo, pero esta vez entendidos de una manera diferente. Como en el caso anterior, los objetos existen realmente, pero existen en relación con la aplicación de un marco conceptual que tampoco puede ser considerado absoluto.

27 Así, la experiencia que tenemos con el mundo está constituida por nuestros conceptos, no podemos conocer nada del primero al margen de los segundos. El mundo existe para nosotros y lo entendemos sólo desde un punto de vista humano, “[...] los 'objetos' mismos son tanto construidos como descubiertos, tanto productos de nuestra invención conceptual como del factor 'objetivo' en la experiencia [...]" (Putnam 1981: 54). Donde el sentido de "objetivo" se entiende como construcción intersubjetiva, no como correspondencia metafísica.

Hemos dicho que los sujetos entienden al mundo desde alguno de los diversos marcos conceptuales posibles. Estos marcos no son absolutos, no son fijos ni establecidos definitivamente, sino que son construcciones humanas que pueden cambiar. Los sujetos de una comunidad no están irremediablemente constreñidos a un único marco conceptual sino que los sujetos pueden llegar a relacionarse con otros marcos conceptuales y descubrir que, como afirma Sosa, "lo que existe en relación con un esquema conceptual puede no existir en relación con otro" (Sosa 1992: 79). Pero, volvemos a insistir, los marcos conceptuales no se construyen al margen del mundo, sino en relación con éste, lo que llamamos mundo es tanto un mundo conceptual como real constituido desde las prácticas sociales. De acuerdo con Goodman, cuya postura nos parece compatible con el realismo interno, sostenemos que "[...] no hablamos ahora 
de múltiples alternativas posibles a un único mundo real, sino, por el contrario, de múltiples mundos reales [...]" (Goodman 1990: 18-9).

Dewey, por ejemplo, explica la tradición filosófica presente hasta sus días, la cual estaba comprometida al menos con dos tesis:

a) Que el objeto de conocimiento es alguna forma de Ser último que precede a la investigación reflexiva, así como a toda función del conocer humano, y es independiente de ellos.

b) Que este ser antecedente posee entre las características que lo definen, propiedades que pueden ejercer autoridad en la formación de nuestros juicios de valor, es decir, rige sobre los fines o propósitos que han de guiar nuestra conducta.

Desde esta perspectiva, la filosofía tendría como finalidad el conocimiento de ese Ser y de sus propiedades esenciales. Esta concepción implicaría que el conocimiento humano se explica en el marco de una teoría "copia" de lo real, entendiendo por "real" aquello que existe de un modo estructurado, fijo e independiente de nuestra actividad de conocer. Una postura como ésta busca una certeza absoluta, lo que implica la separación entre teoría y práctica, entre conocimiento y acción. En la propuesta de Dewey, el esquema recién descrito es inaceptable, y se compromete, en cambio, con la tesis de vinculación entre conocimiento y acción.

Dewey se pregunta reiteradamente a lo largo de su obra, si está justificada la teoría según la cual el conocimiento es válido en la medida en que representa una descripción de existencias independientes de toda actividad humana, y también se pregunta si está justificada la doctrina que sostiene que los fines y valores son asimismo independientes de la actividad humana. Nuestro autor da una respuesta negativa y se afirma en una postura crítica a la tradición del modelo racionalista teleológico (Di Gregori y Durán 2004-2005: 2-5).

\section{Verdad, realidad y justificación}

La idea del plan cooperativo al que alude Dewey en relación con la dimensión política se vincula con la búsqueda de las condiciones en las que la Gran Sociedad se pueda transformar en la Gran Comunidad. Para este pensador, la idea de una comunidad se corresponde con fases reales de la vida asociada donde los sujetos se hallan libres de elementos restrictivos. Donde hay una actividad conjunta cuyas consecuencias se juzguen adecuadas por las personas que intervienen en ella, y donde la consecución de ese bien produzca un deseo firme y un esfuerzo decidido por conservarlo como un bien compartido, se puede hablar de una vida comunitaria y democrática (Dewey 2004b: 137-8).

Pero las prácticas agregadas de los sujetos no constituyen por sí mismas una comunidad, es necesario participar en la formulación y negociación de algunos fines que se compartan e interesen a los actores involucrados. La reflexión moral emergerá cuando los hombres confronten situaciones en las que los fines sean incompatibles, aunque estén moralmente justificados. Dicha reflexión moral será necesaria al momento de presentarse un conflicto entre valores que pueden ser igualmente buenos y no obstante, se debe tomar una decisión (Dewey y Tufts 1936: 173-5). Para Dewey, lo que debe prevalecer no son las razones de unos pocos que se impongan a los demás sino 
el proceso activo de pensar, de ahí el carácter indispensable en su propuesta de la democratización del proceso educativo (Di Gregori y Durán 2004-2005: 5).

La experiencia en Dewey es entendida como acción orientada a la resolución de problemas y la elección entre las muchas alternativas posibles a tales problemas, obligan en el ámbito de lo colectivo a que las elecciones racionales requieran cooperación y acuerdo. Pues no hay nada exterior que predetermine universalmente las mejores elecciones. Cada hábito de respuesta y modos de resolver problemas, nos dicen las autoras, ha sido resultado de la práctica interesada de las generaciones antecesoras. El que no haya soluciones predeterminadas permite que podamos reformular y modificar nuestras posturas en vistas de resolver nuevas o antiguas problemáticas (Di Gregori y Durán 2004-2005: 13-4).

Siguiendo las ideas base del pluralismo ontoepistemológico que hemos esbozado, dado que los sujetos constituimos el mundo en el que vivimos desde algún marco conceptual, todos nuestros conceptos, incluidos el de verdad y el de realidad, así como nuestras normas morales y políticas, están ligados a algún marco conceptual. Desde esta postura, las nociones de "justificación" y "verdad" están estrechamente vinculadas. No es posible identificar línea alguna que divida la realidad de aquello que ponen los sujetos cognoscentes, porque la única realidad que existe para un sujeto cognoscente es la que ya constituye desde alguno de los posibles marcos conceptuales. Con respecto a la noción de verdad, a lo único que podemos acceder es a las mejores razones con las que se sustenta la verdad de una creencia, de tal suerte que, con lo que contamos es con nuestras justificaciones. En el mismo tenor, leemos en Dewey su posición respecto a la imbricación entre hechos y valores; la mentada dicotomía entre ambos es falsa. Por otro lado, Ruth Anna Putnam tiene razón al afirmar que los valores orientan nuestras razones para tomar decisiones, y en este sentido, guían nuestras acciones. Para poder decidir, los sujetos apelan a los valores que provee el marco existente (Putnam 1985: 199). Así pues, el pluralismo de valores no puede dejar de afectar y de guiar nuestras justificaciones en el ámbito epistemológico, ético y político.

La relevancia del pluralismo ontoepistemológico, así como de las respectivas posturas epistemológicas de James, Dewey y Rorty que presentamos, reside en que si bien la noción de verdad está estrechamente ligada a la de justificación, no por ello cualquier razón puede ser considerada como válida para sostener la verdad de una creencia o para sustentar lo acertado de una toma de decisión personal, colectiva o política, como de hecho sucede en el caso de las condiciones de la llamada "posverdad." Un ejemplo lo tenemos en las razones subjetivas, insuficientemente justificadas, con base en las cuales una cantidad creciente de padres de familia, en el ámbito internacional y guiados por la idea de "posverdad," ha decidido no vacunar a sus hijos contra el sarampión, paperas y rubeola (MMR) por sus siglas en Inglés. Las razones que aducen los padres de familia son que este coctel de vacunas para las enfermedades indicadas podría causar autismo a sus hijos.

37 Según Collins y Pinch lo que se sabe hasta el momento es que un número de niños comienza a mostrar síntomas de autismo en los primeros años de vida, aproximadamente en el tiempo en el que también es administrada la (MMR). En los casos en los que el autismo se presenta después de la vacunación, los padres sostienen con base en razones subjetivas, que hay una secuencia temporal como si fuera la causa del autismo en los niños. En cambio, el hecho comprobado y bien documentado con base en buenas razones de que si sus hijos enfermaran de sarampión podrían tener daño 
cerebral, carece de fuerza. La primera información de una relación entre autismo y vacunación nos dice Collins, proviene de las preocupaciones de los padres de familia antes que de investigación científica reportada (Collins \& Pinch 2005: 181-2).

El hecho de que en la actualidad haya un número creciente de casos de sarampión en diferentes países que habían sido declarados libres de esta enfermedad, muestra que los padres de familia tienen por verdaderas creencias falsas que les guían a tomar decisiones erradas. Los padres de familia ignoran las razones válidas en las que se sustenta un conocimiento y optan por justificar sus creencias desde los motivos más personales.

39 La postura pluralista que discutimos aquí en relación con una noción de verdad estrechamente relacionada con una noción de justificación, adquiere importancia porque muestra que no toda justificación es válida, se requiere cuestionar, revisar, volver a indagar si las razones que sustentan nuestras creencias, así como las acciones que éstas guían, son acertadas y no propician unas condiciones de daño. La formulación de nuestras hipótesis y el ponerlas a prueba, confrontándolas con otras hipótesis relacionadas con otros marcos conceptuales, tendría como finalidad investigar si se trata de hipótesis que comprueban ser acertadas o no. Esta manera de proceder nos permite constrastar y poner a prueba alternativas diferentes a nuestras prácticas sociales y creencias; en la experiencia se verá si son acertadas o se refutan, pero si se refutan, como es el caso de la creencia de que la aplicación de la (MMR) causa autismo, debe reconocerse que se invalida dicha justificación.

En esta línea, retomamos a Rorty quien subraya que si hay algo distintivo en el pragmatismo, es que apoya la noción de un futuro humano mejor. De acuerdo con Dewey, en su texto sobre la influencia del Darwinismo en la filosofía, el principio de selección natural y, en términos generales, la lógica darwiniana, genera cambios como el paso de la idea de un propósito fijo relacionado con una esencia hacia la idea de que hay cambios específicos relacionados con propósitos concretos. También se pasa de la idea de una inteligencia que da forma a las cosas de una vez y para siempre, a la posición de que hay inteligencias particulares de objetos que siguen transformándose. Hay un cambio desde la idea de una última meta o de una causa trascendente que nos conduce a "El Bien," hacia avances directos y concretos relacionados con acciones justas. Con el Darwinismo se deja de creer en la validez de ciertas preguntas y, lejos de resolverlas o responderlas, las superamos para formular otras preguntas en relación con nuevos y diferentes contextos que están en transformación continua (Dewey 1910: 1-19). Siguiendo a Dewey y a Darwin, Rorty señala que lo único que justifica una mutación, biológica o cultural, es su contribución a la existencia, en algún lugar del futuro, de una especie más compleja e interesante. La justificación es siempre una justificación desde el punto de vista de los sobrevivientes (Rorty 1997: 13-4). En el ejemplo señalado sobre la aplicación de vacunas, la mejor justificación estaría ofreciendo una garantía a la medida humana de asegurar las mejores condiciones posibles de salud a los menores.

41 Los pragmatistas no creen que haya una manera en la que las cosas realmente son, al margen de nuestras justificaciones. Por ello, buscan reemplazar la distinción apariencia-realidad por una distinción que podríamos interpretar como que hay justificaciones menos útiles y justificaciones más útiles; útiles para crear un futuro mejor (Rorty 1997: 14-5, 18). 
Donald Davidson por ejemplo, relaciona la noción de verdad con la posibilidad de comunicación entre hablantes e intérpretes. Una base común para interpretar exitosamente nuestros respectivos mensajes implicaría que hay una base conmensurable que se infiere a partir de las intersecciones entre los diferentes mundos $\mathrm{y}$ de las que somos testigos justamente cuando logramos interpretar acertadamente a nuestro interlocutor, esto es, cuando efectivamente podemos comunicarnos. Queda pendiente si podríamos afirmar, o no, que es posible la comunicación entre personas de diferentes esquemas conceptuales con todo y que no podamos comprobar un esquema neutral o un lenguaje neutral. Tampoco se ha podido comprobar que haya un esquema único o una ontología común que compartamos y a la que accedamos al margen de cualquier esquema conceptual. Para Davidson la verdad de las oraciones sigue siendo relativa al lenguaje, y esto es todo lo objetivo que podemos ser. Al renunciar al dualismo de esquema y mundo no renunciamos al mundo, sino que restablecemos el contacto inmediato con los objetos familiares en relación con los cuales nuestras oraciones y opiniones son verdaderas o falsas (Davidson 1984). La idea de que el contenido proposicional de las oraciones de observación se determina mediante lo que es común y sobresaliente al hablante y al intérprete tiene consecuencias importantes para la relación entre el pensamiento y el significado, y para nuestra concepción del papel de la verdad.

Porque no sólo asegura que hay un nivel básico en el cual los hablantes comparten sus concepciones, sino que también que lo que ellos comparten es una visión ampliamente correcta de un mundo común. La fuente última tanto de la objetividad como de la comunicación es el triángulo que, al relacionar hablante, intérprete, y el mundo, determina los contenidos del pensamiento y del habla. Dada esta fuente, no hay lugar para un concepto relativizado de verdad. (Davidson 1997: 85)

Efectivamente, una proposición que es verdadera en relación con un lenguaje, desde un marco conceptual, no puede ser el caso de que pudiendo formularse esa misma proposición en relación con otro marco conceptual, sea falsa. Desde la postura del pluralismo ontoepistemológico expuesto, si bien las razones con las que se sustenta la verdad de una creencia son relativas a un marco conceptual, esto no significa que sean arbitrarias, no cualquier razón que se aduzca y que se considere adecuada es una razón válida.

Uno de los esfuerzos que hicieron James y Dewey fue buscar la manera de reemplazar la tarea de justificar la costumbre y la tradición pasadas que apelaban a una estructura inalterable, por la tarea de reemplazar un presente insatisfactorio por un futuro más satisfactorio. En muchas ocasiones un criterio para validar las razones que sustentan una creencia es su coherencia con las creencias que la anteceden. Sin embargo, esto sólo muestra la firme adhesión a ciertas creencias, a un marco conceptual específico y a una forma estipulada de justificar la verdad de esas creencias, pero no dice nada más.

Así, siguiendo a Dewey, deberíamos dejar de lado la idea de que el conocimiento es el intento de representar la realidad, y en cambio, habría que visualizar la indagación como un modo de usar la realidad. La relación entre nuestras alegaciones de la verdad y el resto del mundo es causal más que representacional, es causa de que sostengamos creencias (Rorty 1997: 21-9).

No hay manera de mostrar que cuanto mejor justificada esté una creencia es más verosímil que sea verdadera. Una creencia justificada con base en razones válidas lo que muestra es que en ese momento histórico no hay razón pública y accesible que la contravenga, pero eso no elimina la posibilidad de que en un futuro, frente a otras 
audiencias, se encuentren razones que socaven la validez de la creencia, y con ello, se muestre que tal creencia no estaba suficientemente justificada aunque así se creyera durante mucho tiempo atrás. Es decir, una creencia puede considerarse verdadera porque hasta el presente no hay razones que contravengan su correcta justificación, pero dado que no hay garantía absoluta de que en un futuro esas razones no serían rechazadas como buenas razones, no podemos afirmar que una creencia esté en definitiva anclada a la verdad o a la realidad independientemente de nuestras razones. No hay conexión entre verdad y justificación, confundimos el hecho de que al estar mejor justificadas las creencias sean verdaderas, pero no podemos hacer una afirmación como ésta, pues nos comprometería a aceptar que hay algo externo a nuestras justificaciones llamado "La Verdad" y la pregunta que se sigue es ¿Cómo sabemos que existe algo llamado "Verdad" si no podemos tener la perspectiva del Ojo de Dios?

Puede parecer extraño sostener que no hay conexión entre nuestras justificaciones y la verdad, el problema es que, como indica Rorty, somos propensos a considerar que la verdad es la meta de la indagación.

La indagación y la justificación son actividades en las que nosotros, usuarios del lenguaje, no podemos menos que involucrarnos. No necesitamos una meta denominada "verdad" que nos ayude a eso, así como los órganos digestivos no necesitan una meta llamada "salud" para comenzar a funcionar. Los usuarios del lenguaje no pueden menos que justificar sus creencias y deseos, unos con otros, tal como los estómagos no pueden evitar procesar la comida. La agenda de nuestros órganos digestivos es establecida por los alimentos específicos que procesan y la agenda de nuestra actividad justificatoria es proporcionada por las distintas creencias y los diferentes deseos que encontramos en los demás usuarios del lenguaje. Sólo habría una meta "superior" de la indagación, llamada "verdad," si hubiera una cosa tal como la justificación última, una justificación ante Dios o ante el tribunal de la razón, en tanto distintos de cualquier audiencia meramente finita. (Rorty 1997: 35)

No puede haber un tribunal así, tendría que tomar en cuenta todas las alternativas a una creencia dada y conocer todo lo que fuera pertinente para criticar cada una de esas alternativas. Ese tribunal tendría que tener una perspectiva (metafísica) que tomara en cuenta todos los rasgos del mundo en tanto descrito por un conjunto dado de términos, además de cada rasgo desde toda descripción posible.

Un miembro pleno de su comunidad, nos dice Rorty, estará en condiciones de producir una justificación para la mayoría de sus creencias, una justificación que satisfaga los requerimientos de la comunidad.

No hay, sin embargo, ninguna razón para pensar que las creencias en mejores condiciones de justificarse sean las más probablemente verdaderas, ni que las menos justificadas sean las que con más probabilidad resulten falsas. El hecho de que la mayoría de las creencias sean justificadas, como el hecho de que la mayoría de las creencias sean verdaderas, es una mera consecuencia más del carácter holista de la adscripción de creencias. (Rorty 1997: 34)

50 James nos recuerda que la ciencia y la religión son ambas vías respetables para adquirir creencias respetables, no obstante tratarse de creencias que son buenas para propósitos muy diferentes. Lo que existe es sencillamente, el proceso de justificar las creencias ante una audiencia. Y ninguna audiencia está más próxima a la naturaleza ni es mejor representante que cualquier otra de algún ideal ahistórico de la racionalidad (James 
2003). Peirce nos diría que las creencias son reglas para la acción más que intentos para representar la realidad (Rorty 1997: 32).

La verdad no es algo hacia lo que nos dirigimos, ni es algo a lo que nos aproximamos más cuanto más justificación tenemos, no es nuestra meta. No hay nada que afirmar sobre los límites del conocimiento ni nada que decir respecto a la conexión entre justificación y verdad, el único sentido que tiene contrastar lo verdadero con lo meramente justificado es contrastar un futuro posible con un presente actual, nuestros fines en perspectiva en relación con nuestras condiciones de existencia entendidas como medios. Más específicamente, contrastamos lo verdadero con lo justificado no en lo general sino para contrastar una audiencia actual, con sus requerimientos actuales de justificación, y una esperada audiencia futura (Rorty 1997: 36-7).

La relevancia práctica de nuestras justificaciones desempeña aquí un papel fundamental, James nos habla de que las verdades de las creencias son relevantes porque tienen consecuencias prácticas. La verdad significa su acuerdo con la realidad, así como la falsedad significa su desacuerdo con ella; estar de acuerdo con la realidad significa establecer un contacto funcional que nos permita relacionarnos con ella mejor que si estuviéramos en desacuerdo, a saber, que nuestras creencias y acciones lejos de generar obstáculos fluyen (James 2011: 9-10). En el caso de las vacunas, hasta ahora se puede afirmar que con base en razones válidas funcionan, aciertan según lo previsto. La "funcionalidad" con la que deben contar las creencias para ser verdaderas significa las funcionalidades concretas, físicas o intelectuales, actuales o posibles, que puedan establecer unas con otras en una experiencia concreta (James 2011: 15). El carácter exitoso, acertado o satisfactorio de las acciones y las buenas razones que las justifican es provisional, ninguna de éstas puede funcionar o ser completamente satisfactoria en todos los contextos y en cualquier tiempo histórico. Dado que nadie conoce el futuro, nadie sabe qué creencias permanecerán o no justificadas, no hay nada ahistórico que decir acerca del conocimiento o de la verdad (en general) (Rorty 1997: 40).

Según Dewey, cuando pensamos en el conocimiento y la verdad, el punto más importante que podemos recoger de Darwin es que no hay un orden natural de las razones para justificar una creencia, como no hay un orden natural para la evolución biológica. Sólo podría existir un orden tal si todas las alternativas futuras ya estuvieran presentes. Una posición como ésta querría reconocer los límites de la posibilidad, es decir, de la investigación posible, del conocimiento posible, de las formas posibles de vida humana (Dewey 1910: 1-19). La evolución cultural, la evolución de las demandas de justificación, no se produce de acuerdo con leyes, así como no se produce de acuerdo con leyes la evolución biológica, es decir, la evolución de las demandas de espacio vital. Ambas se producen por una secuencia fortuita de acontecimientos, algunos afortunados y otros no (Rorty 1997: 40-1).

\section{Conclusiones}

En este trabajo hemos sostenido como idea principal el hecho de que no es posible ningún conocimiento al margen de algún marco conceptual y que las razones en que se base, por válidas que sean, no nos permiten reconocer la verdad como distinta de la justificación. 

llamado realismo interno de Putnam, por pragmatistas como Dewey y James y por el pluralismo onto-epistemológico expuesto. Desde la postura epistemológica defendida, afirmamos que dado que sólo contamos con nuestras justificaciones y no hay manera de que podamos acceder a la verdad o a la realidad al margen de ellas, la formulación de nuestras hipótesis y el ponerlas a prueba, confrontándolas con otras hipótesis relacionadas con otros marcos conceptuales, no debería tener como fin comprobar una verdad o restricción de la realidad, sino más bien mostrar que se trata de una hipótesis que comprueba ser útil, acertada, que funciona, en unas condiciones de existencia específicas. Esta posición nos permite dejar de preocuparnos por la verdad o la realidad en un sentido metafísico, y en su lugar, confrontar nuestra postura con alternativas diferentes a nuestras prácticas sociales, justificaciones y creencias; en la experiencia se verá si son acertadas o se refutan. Hemos ejemplificado con el caso de un número cada vez mayor de padres de familia que opta por no vacunar a sus hijos; una decisión errada, sustentada en razones insuficientes, como suele ocurrir con posturas como la llamada "posverdad," causando con ello el aumento sistemático de casos en el mundo de personas enfermas de sarampión.

Wittgenstein explica que lo que hace a una proposición verdadera y que la prueba de la misma sea vinculante depende de lo que hacemos, de cómo la usamos. En palabras de Wittgenstein: "procedo a través de la prueba, y luego acepto su resultado. - Quiero decir: esto es simplemente lo que hacemos" (Wittgenstein 1967: 20 \$63). A este respecto Pitkin explica que la prueba debe ser tal que tiene que mostrarnos que algo es así, pero también cómo es así, (Pitkin 1984: 346) "no aceptamos el resultado de una prueba porque resulte una vez, o porque resulte a menudo. Pero vemos en la prueba la razón de decir que esto tiene que ser el resultado" (Wittgenstein 1967: 81 \$39). cómo es aceptable (Wittgenstein 1967: 22 §75). En este sentido, decimos que las buenas razones pueden considerarse como una guía a la medida humana que garantiza a los sujetos que sus prácticas sociales no son arbitrarias.

Si aceptamos que las nociones de realidad y de verdad están estrechamente ligadas a la noción de justificación, podríamos considerar que, como explica Pitkin, allí donde algo no puede ser modificado con base en buenas razones, se hace sospechoso el continuo deseo de cambio (Pitkin 1984: 487). Podríamos decir que la justificación intersubjetiva de las diversas creencias, normas, políticas y toma de decisiones, basada en buenas razones relevantes en algún contexto, hace posible y exige de nosotros que consideremos con seriedad a otras personas, así como el daño que podríamos causar si tomamos decisiones equivocadas y arbitrarias, sustentadas únicamente en razones sobre las que se ha indagado poco; que escuchemos realmente, que lleguemos a ser capaces de comprender desde la perspectiva del otro. También hace posible, y nos exige, que nos tomemos seriamente a nosotros mismos. Esto no significa que no podamos cambiar en algún grado nuestros conceptos, nuestras prácticas o nuestras instituciones, significa que no todo cambio es posible. Es decir, no todo cambio está suficientemente justificado, donde la validación de una afirmación dependerá de sus consecuencias, Schiller (1907:183) y deberá considerarse en relación con los motivos razonables que influyen en la deliberación. 


\section{BIBLIOGRAFÍA}

CAVELl Stanley, (1979), The Claim of Reason, Oxford, Oxford University Press.

COLLINS Harry \& Trevor PINCH, (2005), Dr. Golem. How to think about medicine, Chicago, The

University of Chicago Press.

DAVIDSON Donald, (1984), On the Very Idea of a Conceptual Scheme. Inquiries into Truth and

Interpretation, Oxford, Clarendon Press.

DAVIDSON Donald, (1997), Estructura y contenido de la verdad, Madrid, Tecnos.

DEWEY John, (1950), Lógica. Teoría de la investigación, México, Fondo de Cultura Económica.

DEWEY John, (2004a), Democracia y Educación, Madrid, Ediciones Morata.

DEWEY John, (2004b), La opinión pública y sus problemas, Madrid, Ediciones Morata.

DEWEY John (2007), The Influence of Darwin on Philosophy, New York, Henry Holt and Company.

DEWEY John, (2008), Teoría de la valoración, Madrid, Siruela.

DEWEY John, (2010), Experiencia y Educación, Madrid, Biblioteca Nueva.

DEWEY John \& James H. TUFTS, (1936), Ethics, Nueva York, Henry Holt and Company.

DI GREGORI María Cristina \& Cecilia DURÁN, (2004-2005), “Conocimiento y acción: una concepción

fundante para el modelo pedagógico y político de John Dewey," Educación Física y Ciencia 7, 55-67.

[En línea] Disponible en: [fuentesmemoria.fahce.unlp.edu.ar/art_revistas/pr.106/pr.106.pdf].

GOODMAN Nelson, (1990), Maneras de hacer mundos, Madrid, Visor.

HABERMAS Jürgen, (1998), Facticidad y validez sobre el derecho y el Estado democrático de derecho en términos de teoría del discurso, Madrid, Trotta.

JAMES William, (2000), Pragmatismo. Un nuevo nombre para viejas formas de pensar, Madrid, Alianza.

JAMES William, (2011), El significado de la verdad. Una secuela de Pragmatismo, Barcelona, Marbot Ediciones.

JAMES William \& William K. CLIFFORD, (2003), La voluntad de creer. Un debate sobre la ética y de la creencia, Madrid, Tecnos.

KUHN Thomas S., (2000), “The Road Since Structure," in J. Conant \& J. Haugeland (eds), The Road Since Structure. Philosophical Essays, 1970-1993, Chicago, University of Chicago Press, 90-104.

OLIVÉ León, (1996), Razón y sociedad, México, Fontamara.

OLIVÉ León, (1999), Multiculturalismo y pluralismo, México, Paidós-UNAM.

PÉREZ Ana Rosa, (1993), “El realismo de Villoro,” in E. Garzón \& F. Salmerón (eds), Epistemología y cultura en torno a la obra de Luis Villoro, México, UNAM, 39-62.

PITKIN Hanna Fenichel, (1984), Wittgenstein: El lenguaje, la política y la justicia. Sobre el significado de Ludwig Wittgenstein para el pensamiento social y político, Madrid, Centro de Estudios Constitucionales.

PUTNAm Hilary, (1981), Reason, Truth, and History, New York, Cambridge University Press. 
PUTNAM Hilary, (1990), Representación y realidad. Un balance crítico del funcionalismo, Barcelona, Gedisa.

PUTNAm Hilary, (1994), Las mil caras del realismo, Barcelona, Paidós-I.C.E.-U.A.B.

PUTNAM Hilary, (1997), La herencia del pragmatismo, Barcelona, Paidós.

PUTNAM Ruth Anna, (1985), “Creating Facts and Values,” Philosophy, 60 (232), 187-204.

QUINTANILla Miguel Ángel, (1994), "Introducción," in H. Putnam (A.), Las mil caras del realismo, 17-35, Barcelona, Paidós-I.C.E.-U.A.B.

RORTY Richard, (1997), ¿Esperanza o conocimiento? Una introducción al pragmatismo, Buenos Aires, Fondo de Cultura Económica.

RORTY Richard, (2000), El pragmatismo, una versión. Antiautoritarismo en epistemología y ética, Barcelona, Ariel Filosofía.

SCHILLER Ferdinand Canning Scott, (1907), Studies in Humanism, London, New York, Macmillan and Co. Limited.

sosA Ernesto, (1992), “El realismo pragmático de Putnam," Diánoia Anuario de Filosofía, XXXVIII (38), 63-84.

WITTGENSTEIN Ludwig, (1967), Remarks on the Foundations of Mathematics, Oxford, Basil Blackwell.

\section{RESÚMENES}

In this article I argue that the notion of truth is so closely linked to the notion of justification that it is not possible to access the truth, even with the best reasons upon which a justification is supported.

I'll show that the notions of truth and reality can only be inferred from our experience of the world, in which our social practices can be verified or disproved, and if our hypotheses are accepted or refuted. I maintain that we can only access realities which we constitute in relation to one of several possible conceptual frameworks. These conceptual frameworks are not transcendental entities nor are they established definitively, they are social constructs. This means that the conceptual frameworks are constituted by the social practices of the subjects. We understand that social practices are institutionalized groups of actions undertaken by members of a community and directed toward the consecution of an end. These actions presuppose that the beliefs, norms and values are shared by members of an epistemic community. Olivé (1999: 135-42).

This article begins by presenting Rorty's counter-proposal for the notion of truth, in relation to which I put forward the principal theses of onto-epistemological pluralism. Subsequently, I develop the idea of inter-subjective contextual justification and I show that this posture, although it does depend on specific conditions for justification, is not arbitrary.

I demonstrate the relevance of onto-epistemological pluralism and the need to adequately substantiate our beliefs and decision making, by presenting the case of parents guided by a notion of 'post-truth' who decide not to vaccinate their children against measles based on insufficiently justified subjective reasons. A fact that is having serious harmful consequences to health, particularly among young children. 


\section{AUTOR}

MÓNICA GÓMEZ SALAZAR

PhD. Facultad de Filosofía y Letras, Colegio de Filosofía. Universidad Nacional Autónoma de México

monigomi73[at]yahoo.com 